\title{
La depresión es un factor de riesgo coronario en hombres
}

Depression in a risk factor for coronary artery disease in men. The Precursors Study.

Ford DE, Mead LA, Chang PP et al. Arch Intern Med 1998.58:1422-26.

\section{Objetivo}

Determinar si la depresión es un factor de riesgo independiente para el desarrollo de enfermedad coronaria.

\section{Diseño}

Estudio poblacional de cohorte, prospectivo, con una media de seguimiento de 37 años.

\section{Lugar}

Facultad de Medicina de la Universidad Johns Hopkins, Baltimore, EE.UU.

\section{Participantes}

Se evaluaron a 1190 varones, con edad promedio de 26 años a través del examen físico, los antecedentes familiares y personales. El seguimiento fue con encuestas anuales enviadas por correo. Se excluyeron los casos con información incompleta, depresión al inicio del estudio, e imposibilidad de seguimiento.

\section{Evaluación de los factores de riesgo}

Se evaluó colesterol, tensión arterial, diabetes, sedentarismo, tabaquismo, y antecedentes familiares de infarto de miocardio (IAM) prematuro.

\section{Medición de resultados principales}

La depresión fue determinada a través de autocuestionarios, y luego evaluada por un comité, descartándose los casos con síntomas menores a 2 semanas, y los relacionados con duelo. Los eventos coronarios (verificados con las historias clínicas) fueron: IAM, angi- na de pecho, by-pass o angioplastia; los eventos cardiovasculares fueron: insuficiencia cardíaca, patología cerebrovascular, hipertensión arterial e insuficiencia renal.

Se analizaron como variables, además de la depresión, hipertensión arterial, diabetes, dislipemias, y consumo de cigarrillo, café y alcohol. Se excluyeron los casos de cardiopatía acontecidas durante los 2 primeros años de la depresión.

\section{Resultados Principales}

La edad promedio de la cohorte estudiada fue de 66 años. La incidencia acumulativa de depresión fue del $12 \%$, con un promedio de 46 años para el primer episodio. No tuvieron tratamiento un $23 \%$, usaron antidepresivos un $33 \%$, y realizaron psicoterapia con o sin psicofármacos un $44 \%$. La depresión se asoció con el doble del riesgo para eventos coronarios, y este no varió al ajustarse por los factores de riesgo tradicionales (colesterol, tabaquismo, hipertensión, y diabetes), índice de masa corporal, y antecedentes familiares de IAM, siendo su asociación estadísticamente significativa (RR=1.7, IC95\% 1.0 a 2.9).

En el análisis multivariado, la depresión tuvo un $\mathrm{RR}=2.12$ para coronariopatía (IC95\% 1.24-3.63) y tambien de 2.12 para IAM (IC95\% 1.114.06). El tiempo promedio entre el primer episodio de depresión y el evento cardiovascular fue de 15 años.

\section{Conclusiones}

En hombres, la depresión esta asociada con un aumento del riesgo de enfermedad coronaria, independientemente de los factores de riesgo tradicionales.

\section{COMENTARIO}

Los estudios epidemiológicos basados en los criterios diagnósticos del DSM III, detectaron un $11 \%$ de depresión en la población adulta.

La depresión aumenta más de 4 veces el riesgo para IAM ${ }^{1}$, con un $R R=1.5$ para mortalidad por coronariopatía. Constituye un factor de riesgo independiente para enfermedad coronaria, y no se relaciona con otros trastornos psiquiátricos tales como fobias o estados de ansiedad, ni con el uso de los antidepresivos tricíclicos o benzodiazepinas ${ }^{1}$; pero sí con litio, fenotiazinas y meprobamato. Estudios posteriores, como el National Health Examination Follow-up Study ${ }^{2}$, determinaron que los estados depresivos y de desesperanza, y no sólo la depresión mayor, se asocian a mayor riesgo de IAM, lo cual se mantiene luego del ajuste por los factores de riesgo tradicionales (edad, sexo, tabaquismo, hipertensión arterial, y diabetes), enfermedades preexistentes, y variables socio-demográficas, tales como raza, educación, estado marital y consumo de alcohol.

En la depresión subyacen varios trastornos que facilitarían los eventos coronarios, como el aumento de catecolaminas y cambios en la función autonómica, pudiendo llegar a aparecer arritmias ventriculares; injuria endotelial, aumento en la agregación plaquetaria ${ }^{2}$, y resistencia periférica a la insulina, lo cual facilitaría el desarrollo de la arterioesclerosis ${ }^{2-4}$. Además existe una relación entre los estados depresivos y el hábito tabáquico, ya que la nicotina podría aliviar los estados disfóricos, activando algunos neurotransmisores centrales; la deprivación de la misma empeoraría los síntomas de depresión ${ }^{3}$.

El presente estudio parece coincidir con los conocimientos actuales acerca de depresión y riesgo coronario, pero merece algunas críticas. En primer lugar *Ver Glosario

\section{Dra. Yvette Morón Hessling}

Clínica Médica.

\section{Referencias}

1. Pratt LA, Ford DE, Crum RM y col. Depression, psychotropic medication, and risk of miocardial infarction. Prospective data from the Baltimore ECA follow-up. Circulation 1996;94:3123-29.

2. Anda R. Williamson D, Jones D, y col Depressed affect, hopelessness, and the risk of ischemic heart disease in a cohort of U.S. adults. Epidemiology 1993:4:285-94

3. Anda R, Williamson D, Escobedo LG y col. Depression and the dynamics of smoking. A national perspective. JAMA 1990;264:1541-45.

4. Winokur A, Maislin G, Phillips JL y col. Insulin resistance after oral glucose tolerance testing in patients with major depression. Am J Psychiatry 1998;145:325-30.

5. Robins LN, Helzer JE, Croughan J y col. National Institute of Mental Health Diagnostic Interview Schedule. Arch Gen Pychiatry 1981;38:381-89. no ha sido detallado el tipo de encuestas utilizadas para el diagnóstico de depresión, ni si fueron validadas con un método estándar. Normalmente en los estudios de epidemiología psiquiátrica se usan modelos diagnósticos validados con entrevistas clínico-psiquiátricas estructuradas, tales como el Diagnostic Interview Schedule (DIS) y otros ${ }^{5}$. Tampoco se basaron en los criterios diagnósticos del DSM III ni anteriores, lo cual deja la impresión de criterios poco comparables. Si bien descartaron los casos con depresión al inicio del estudio, es posible que algunos no hallan sido detectados por el cuestionario inicial. En cuanto al uso de antidepresivos, no diferencian los distintos tipos de drogas; esto es relevante, ya que algunas como litio, fenotiazinas y meprobamato, aumentan el RR de IAM ${ }^{1}$. No están discriminados posibles confundidores de depresión, tales como fobias, ataques de pánico, o consumo de drogas. En relación al análisis estadístico, cuando la depresión se ajusta por factores de riesgo coronario, el RR de eventos coronarios baja de 2 a 1.7, con un IC $95 \%$ que llega hasta el valor 1, lo que en este caso implicaría que la depresión podría no aumentar el riesgo de eventos coronarios.

Finalmente hay que considerar que la cohorte estudiada estaba compuesta sólo por hombres, con un nivel sócioeconómico de medio a alto, siendo por lo tanto poco extrapolable a la población en general.

De todas maneras, más allá de estas observaciones, la evidencia aportada por este estudio prospectivo y la coincidencia de los resultados finales con los de otros estudios ${ }^{1-2-3}$, hace finalmente muy probable que la depresión sea un factor de riesgo independiente para enfermedad coronaria en hombres. Como comenta el editorial de este número de Evidencia, este es otro ejemplo de un estudio donde sólo se incluye a hombres y en el cual las conlusiones son imposibles de extrapolar a las mujeres. 